\title{
Magnetic point contact in ferromagnetic semiconductor (Ga,Mn)As
}

\author{
T. Figielski ${ }^{1}$, T. Wosinski ${ }^{1}$, A. Morawski ${ }^{1}$, O. Pelya, A. Makosa ${ }^{1}$, W. Dobrowolski ${ }^{1}$, J. Wrobel ${ }^{1}$, \\ J. Sadowski ${ }^{2}$, J. Jagielski ${ }^{3}$, J. Ratajczak ${ }^{4}$ \\ ${ }^{1}$ Institute of Physics, Polish Academy of Sciences, 02-668 Warszawa, Poland, \\ e-mail: figiel@ifpan.edu.pl \\ ${ }^{2}$ Institute of Physics, Polish Academy of Sciences, 02-668 Warszawa, Poland and Max-lab, Lund University, 22100 Lund, Sweden \\ ${ }^{3}$ Institute of Electronic Materials Technology, 01-919 Warszawa, Poland \\ ${ }^{4}$ Institute of Electron Technology, 02-668 Warszawa, Poland
}

\begin{abstract}
We show that narrow constrictions, a few hundred nanometers wide, in ferromagnetic-semiconductor (Ga, Mn)As layer exhibit large spin-related magnetoresistance. Moreover, we demonstrate that application of oxygen-ions implantation, instead of chemical etching, is much better method of tailoring nanometer-size circuits in ( $\mathrm{Ga}, \mathrm{Mn})$ As suitable for future spin electronics.
\end{abstract}

Keywords: ferromagnetic semiconductors, magnetoresistance, nanostructures, ion implantation

Paper received 25.11.02; accepted for publication 18.03.03.

\section{Introduction}

Use of the electron spin instead of (or in addition to) the electron charge for the transmitting and processing information is nowadays an ambitious challenge for solidstate scientists. Recently grown GaAs layers containing a few percents of $\mathrm{Mn}$ atoms become ferromagnetic at low temperatures. Despite a rather limited usefulness of lowtemperature ferromagnetics in practice, they might serve as model materials suitable for experiments with nano-size devices and circuits prospective in future spin electronics.

The aim of the present study was to fabricate and investigate a simple magnetoresistive nanodevice formed by a narrow constriction in the ferromagnetic semiconductor layer, which might be called the magnetic point contact [1] (Fig. 1a). It has been predicted that a boundary between the two ferromagnetic domains is to be locked at the constriction. Then, charge carriers passing through the constriction, which carry also the spin, would be scattered at the boundary Bloch wall. After applying an external magnetic field, which orders the spin alignment in the whole layer, the wall disappears and so will do the relevant resistance of the constriction.

\section{Experimental}

We performed experiments on $50 \mathrm{~nm}$-thick layers of $\mathrm{Ga}_{0.99} \mathrm{Mn}_{0.01}$ As grown by a low-temperature molecular- beam epitaxy on GaAs substrates, which were either semiinsulating or doped to $n$-type. The manganese atoms in the layer give rise to the appearance of both the electron acceptors and localized unpaired spins. The layers became ferromagnetic below a temperature of $17 \mathrm{~K}$, as we found by magnetic susceptibility measurements. The origin of ferromagnetism is likely the Ruderman-KittelKasuya-Yoshida (RKKY) interaction between Mn spins $\left(\mathrm{S}_{\mathrm{Mn}}=5 / 2\right.$ for $\mathrm{Mn}^{2+}$ charge state), mediated by holes $[2,3]$.

At first we fabricated narrow (of the order of a few hundred nanometers) constrictions in the (Ga,Mn)As layers using standard electron-beam lithography and chemical etching (Fig. 1b). Unexpectedly, so-obtained constricted samples, grown on semi-insulating substrate, lost their electrical conductivity at liquid helium temperatures. Probably, charge carriers have been captured in surface states appearing on an extra surface area denuded by the etching. The sample conductance could be, however, induced by illumination of the sample with a light generating electron-hole pairs in the layer. The constricted samples displayed under illumination a large magnetoresistance, whose magnitude varied non-monotonously with the magnetic field (Fig. 2).

Wishing to avoid the effect of vanishing conductance in nanometer-size etched structures, we applied in the following a novel method of tailoring the constriction [4]. We have discovered that an implantation of $(\mathrm{Ga}, \mathrm{Mn}) \mathrm{As}$ layers with oxygen ions both inactivates $\mathrm{Mn}$ acceptors and suppresses ferromagnetism (Fig. 3). 


\section{T. Figielski et al.: Magnetic point contact in ferromagnetic ...}

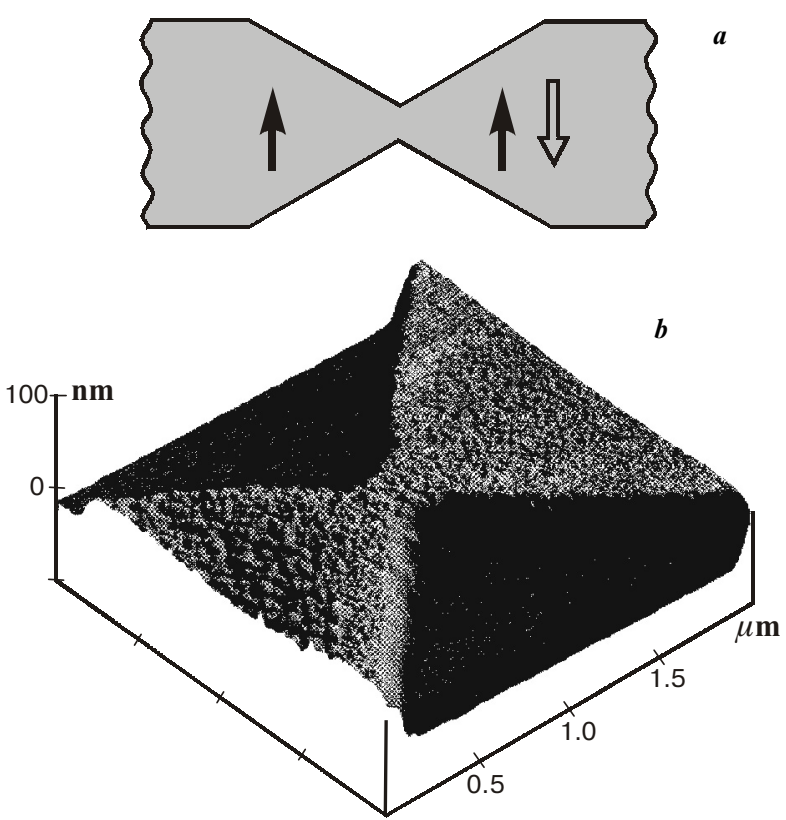

Fig. 1. $a$ - scheme of a constriction showing possible spin orientations in the adjacent ferromagnetic domains with and without magnetic field. $b$ - atomic-force-microscopy image of the constriction fabricated in $(\mathrm{Ga}, \mathrm{Mn}) \mathrm{As}$ layer with use of electron-beam lithography and chemical etching.

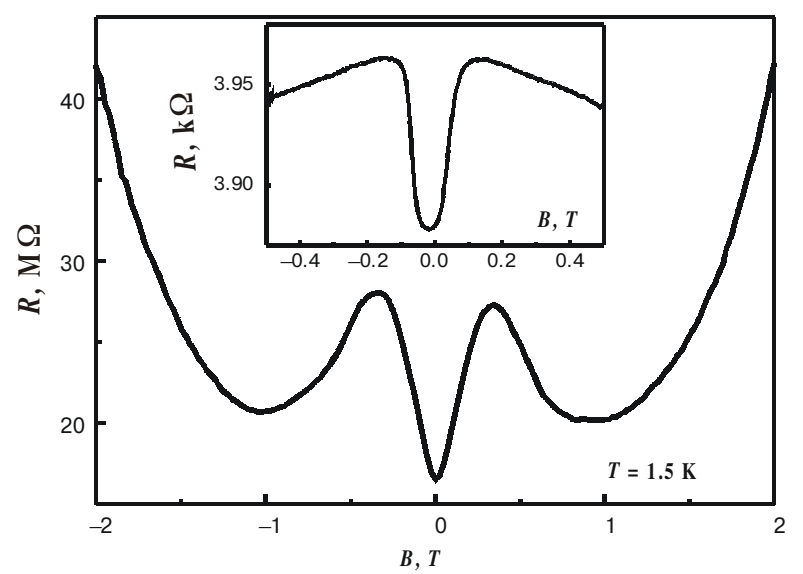

Fig. 2. Resistance of the $\mathrm{Ga}_{0.99} \mathrm{Mn}_{0.01} \mathrm{As}$ constricted sample, fabricated with use of chemical etching, as a function of a magnetic field perpendicular to the layer plane, measured at $1.5 \mathrm{~K}$ under illumination. Inset: same for the reference sample having no constriction.

We implanted oxygen ions of the energy of $25 \mathrm{keV}$ into the $(\mathrm{Ga}, \mathrm{Mn})$ As layer covered with a $500 \mathrm{~nm}$-thick resist (PMMA), on which constriction designs were patterned by means of electron-beam lithography. We applied a dose of $5 \cdot 10^{13} \mathrm{O}^{+}$-ions $/ \mathrm{cm}^{2}$. In accord with our expectations, so-obtained constricted samples were conducting even at liquid helium temperatures. Measure-

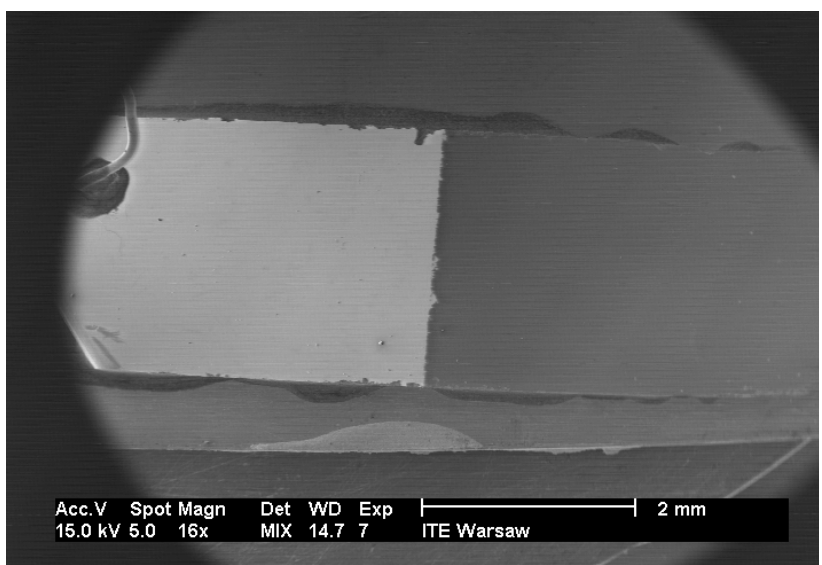

Fig. 3. Micrograph of combined EBIC (electron-beam induced current) and SEI (secondary electron image) in a scanning electron microscope of (Ga,Mn)As layer grown on $n$-type GaAs substrate. The right-hand half of the sample, which does not display an EBIC contrast, has been subjected to oxygen ion implantation.

ments, being presently performed, show an intriguing behavior of the magnetoresistance in the samples obtained in this way.

\section{Conclusions}

The principal result obtained in this study is that the lowfield magnetoresistance of the constricted samples is more than one order of magnitude larger than that of the reference sample without constriction. Our investigations demonstrate also that the oxygen ion implantation might be a prospective method for the fabrication of microcircuits in future spin electronics based on III-V semiconductors containing manganese. The essence of this method - inactivation of $\mathrm{Mn}$ acceptors by the implanted oxygen ions is not fully understood yet.

\section{References}

1. H. Imamura, N. Kobayashi, S. Takahashi and S. Maekawa, Conductance quantization and magnetoresistance in magnetic point contacts // Phys. Rev. Lett. 84, 1003-1006 (2000).

2. H. Ohno, A. Shen, F. Matsukura, A. Oiwa, A. Endo, S. Katsumoto and Y. Iye, (Ga,Mn)As: A new diluted magnetic semiconductor based on GaAs // Appl. Phys. Lett. 69, 363365 (1996).

3. F. Matsukura, H. Ohno, A. Shen and Y. Sugawara, Transport properties and origin of ferromagnetism in $(\mathrm{Ga}, \mathrm{Mn}) \mathrm{As} / /$ Phys. Rev. B 57, R2037-2040 (1998).

4 T. Figielski, T. Wosinski, A. Morawski, O. Pelya, J. Sadowski, A.L. Toth and J. Jagielski, Microcircuit tailoring in ferromagnetic semiconductor $(\mathrm{Ga}, \mathrm{Mn}) \mathrm{As}$, Phys. Stat. Sol. (a) 195, 228-231 (2003). 